\title{
Evaluation Numérique en Didactique du Français Langue Etrangère*
}

\section{Digital Evaluation in Teaching French as a Foreign Language}

\author{
Ahmet AYCAN ${ }^{1}$, Yaprak Türkân YÜCELSIN TAŞ ${ }^{2}$
}

\begin{abstract}
${ }^{I}$ Dr. Öğr. Üyesi, Fransız Dili Eğitimi, Eğitim Fakültesi, Ondokuz Mayıs Üniversitesi, Türkiye, ahmet.aycan@omu.edu.tr, (https://orcid.org/0000-0003-1202-5654)

${ }^{2}$ Doç. Dr., Fransız Dili Eğitimi, Ĕgitim Fakültesi, Marmara Üniversitesi, Türkiye, tyucelsin@marmara.edu.tr, (https://orcid.org/0000-0003-2963-1757)
\end{abstract}

Geliş Tarihi: 13.03.2021

Kabul Tarihi: 28.10 .2021

\section{RÉSUMÉ}

L'objectif principal de cette étude est d'analyser les effets des outils d'évaluation numérique sur la compétence de production écrite des étudiants en didactique du français langue étrangère. Cette recherche menée conformément au modèle de recherche-action, visait à répondre aux questions suivantes : Quels sont les effets des outils d'évaluation numérique sur le développement de compétence de production écrite des étudiants en français ? Quels sont les rôles, avantages et limites des outils numériques dans le processus d'évaluation ? L'univers et l'échantillon de cette étude, où les méthodes de recherche qualitative et quantitative sont utilisées conjointement, sont composés de 28 étudiants inscrits au cours intitulé Lecture et Ecriture Avancé II dans le Département de l'Enseignement du Français Langue Étrangère de l'Université Ondokuz Mayıs. Dans cette recherche, un pré-test, un post-test et une rubrique d'auto-évaluation de compétence de production écrite ont été utilisés et l'analyse des données obtenues a été interprétée comme des résultats quantitatifs et qualitatifs à l'aide du programme SPSS. Les résultats obtenus de la recherche ont montré que les outils d'évaluation numérique ont apporté une contribution statistiquement significative à l'amélioration de compétence de production écrite. Suite à la comparaison des moyennes des étudiants avant et après les tests, la valeur « $\mathrm{p} » \mathrm{a}$ été calculée comme suit : $000<005$, et cette valeur a montré un résultat significatif en faveur du groupe expérimental.

Mots-clés: Evaluation, numérique, production écrite, platforme numérique, langue étrangère.

Öz

$\mathrm{Bu}$ çalışmanın temel amacı dijital değerlendirme araçlarının yabancı dil olarak Fransızca eğitiminde öğrencilerin yazma becerileri üzerindeki etkilerini incelemektir. Eylem araştırması modeline uygun olarak gerçekleştirilen bu araştırmada şu sorulara yanıt aranmıştır: Dijital değerlendirme araçlarının öğrencilerin Fransızca yazma becerilerinin gelişimi üzerindeki etkileri nelerdir? Dijital araçların değerlendirme sürecindeki rolü, katkıları ve sınırlılıkları nelerdir? Nitel ve nicel araştırma yöntemlerinin bir arada kullanıldığı bu çalışmanın evren ve örneklemini Ondokuz Mayıs Üniversitesi Yabancı Diller Eğitimi Bölümü Fransızca Öğretmenliği Anabilim Dalı'nda İleri Okuma ve Yazma II dersine kayıtlı 28 öğrenci oluşturmaktadır. Araştırmada yazma becerisine yönelik ön test, son test ve öz değerlendirme ölçeği kullanılmış ve elde edilen verilerin çözümlemesi SPSS paket programı kullanılarak hem nicel hem de nitel bulgular şeklinde yorumlanmıştır. Araştırma sonucunda elde edilen bulgulara göre dijital değerlendirme araçlarının öğrencilerin Fransızca yazma becerilerini geliştirmede istatistiksel olarak anlamlı katkılar ortaya çıkardığı görülmüştür. Öğrencilerin ön test ve son test ortalamalarının karşılaştırılması sonucunda P değeri,

\footnotetext{
${ }^{*}$ Cet article est issu de la thèse de doctorat intitulée «Problématique d'évaluation numérique en didactique de langue étrangère» soutenue par Ahmet AYCAN sous la direction de Mme Yaprak Türkân YÜCELSİN TAŞ.
} 
$000<, 005$ olarak hesaplanmış, bu değer deney grubunun lehine anlamlı bir sonuç ortaya çıktığını göstermiştir.

Anahtar Kelimeler: Değerlendirme, dijital, yazma becerisi, dijital platform, yabancı dil.

\section{INTRODUCTION}

Les objectifs primordiaux de l'enseignement/apprentissage d'une langue étrangère (LE) sont de développer et d'améliorer la capacité de savoir-faire linguistique y compris écouter, lire, parler et écrire chez les apprenants. Pour atteindre les objectifs fixés, il faut bien déterminer et préciser la méthode la plus appropriée à suivre lors du processus d'apprentissage d'une langue. L'évaluation fait partie intégrante de ce processus et a pour objectif de contrôler les acquis par rapport à plusieurs normes déterminées par l'enseignant et ainsi de comparer les résultats de l'état initial à l'état final. Pour porter un message concernant la performance des apprenants dans le but d'éviter et de corriger les erreurs commises, il faut examiner et suivre fréquemment leurs performances. A ce stade, l'enseignant doit formuler clairement les moments, les buts et les critères ainsi que les barèmes de l'évaluation.

Pour porter un jugement, prendre des décisions, passer à la séquence suivante dans un parcours de formation et pour recueillir les renseignements sur les points forts et sur les points faibles des étudiants, le feedback joue un rôle primordial. Le feedback permet également de suivre une meilleure progression dans l'interprétation des résultats. Le numérique apporte plusieurs avantages visant à rendre l'apprenant plus actif et autonome. Par le numérique, l'enseignant a la possibilité de donner des feedbacks avant, pendant ou immédiatement suite à l'activité de manière synchrone ou asynchrone. Le feedback fourni par l'enseignant peut soutenir les échanges entre l'enseignant et l'apprenant grâce au numérique.

Cependant, avec le développement des nouvelles technologies de l'information et de la communication (TIC), non seulement la dimension de l'enseignement et de l'apprentissage, mais aussi la dimension de l'évaluation ont accrue. Grâce aux technologies numériques, tous les processus d'évaluation sont exécutés dans des environnements interactifs. A partir de notre recherche bibliographique, nous pouvons constater que les nouvelles technologies sont peu appliquées dans les évaluations.

Les TIC ont de meilleures bénéficies en contexte éducatif en démultipliant des ressources dans le processus d'enseignement/apprentissage des LE. Les établissements scolaires dans le monde entier profitent d'usage des TIC dans leurs systèmes éducatifs afin de faciliter et d'améliorer la qualité de l'enseignement au cours de développement des compétences langagières. "L'intégration pédagogique des TIC à l'université devrait avoir pour but de favoriser, faciliter l'apprentissage. " (Karsenti, Savoie Zajc, \& Larose, 2001, p. 93). Le numérique offre aux enseignants la possibilité de favoriser l'interaction avec les apprenants et de mobiliser les savoirs pour créer un réel continuum en dehors de l'école.

\section{CADRE THEORIQUE}

\subsection{Production Ecrite en Didactique du Français Langue Etrangère}

L'enseignement/apprentissage d'une LE regroupe plusieurs séquences didactiques comme compréhension orale/écrite et production orale/écrite dont l'objectif est de faire communiquer les apprenants en utilisant la langue enseignée dans les situations de communication différentes.

En tant que tâche plus complexe, le fait d'écrire est un acte cognitif dont l'objectif est de représenter des idées en utilisant différents signes graphiques. L'écriture se définit comme ; « une représentation de la langue parlée au moyen de signes graphiques. » (Dubois, J., Giacomo, M., 
Guespin, L., Marcellesi, C., Marcellesi, J.-B., \& Mével, J.-P., 2002, p. 165). Ecrire un texte est la construction d'idées dans une chaîne logique autour d'un thème pour représenter les relations entre les connaissances de l'auteur afin de les transmettre aux lecteurs.

De manière générale un texte se compose de trois séquences ; l'introduction dans laquelle l'auteur dresse un portrait général pour répondre à la question de " pourquoi on écrit ce texte ». La deuxième, le développement dont l'objectif est de transmettre les messages en détails aux lecteurs, d'argumenter des opinions et de les prouver avec les phrases logiques. Et enfin la conclusion dans laquelle l'auteur résume les idées à partir de certaines synthèses en portant des réponses.

En tant qu'activité plus complexe, la production écrite regroupe plusieurs actes et exige différents processus cognitifs. Selon la nature du texte, cette compétence a deux sens " L'écriture est une activité à double nature : la composition du texte a des aspects à la fois sociaux et individuels. » (Glopper, K., Kruiningen, J., \& Hemmen, N., 2014, p. 15). Dans les activités d'écriture, les apprenants suivent des processus semblables à la fois dans la LE et dans la langue maternelle. Les étapes de planification, de rédaction du texte et de révision de la création de texte en langue maternelle sont également compromises dans la production de textes en LE. Pourtant, il est possible de dire que le scripteur a plus de difficultée à produire des textes dans une LE.

Certains de ces raisonnements sont la difficulté d'apprentissage, les différences dans les structures linguistiques, les différences interculturelles, les règles de grammaire, la richesse linguistique de l'apprenant, les transferts négatifs et positifs entre les langues, la compétence de vocabulaire de l'apprenant, etc. Ces facteurs influencent le processus de l'exécution de texte dans une LE de manière positive ou négative.

La connaissance des mots joue un rôle important dans le processus de production de texte. La capacité lexicale de l'apprenant est très importante pour refléter la qualité du texte. De plus, la compétence de production écrite a une structure plus systématique contrairement à la compétence de production orale. « L'écriture, par rapport à l'expression orale, peut être considérée comme un système plus standardisé qui doit être acquis par une instruction spéciale. » (Grabowski, 1996, p. 75). La compétence de production écrite sollicite un processus d'apprentissage distinct. Le scripteur doit constamment traduire ses raisonnements en écriture. A ce stade, trouver le mot le plus approprié, adhérer aux règles de grammaire de la langue cible et exprimer ses pensées de manière cohérente font partie des processus cognitifs sur lesquels un apprenant insiste le plus.

\subsection{Problématique d'Evaluation en Didactique du Français Langue Etrangère}

La dimension d'évaluation joue un rôle important dans le processus d'enseignement/apprentissage d'une LE. Elle ne concerne pas seulement les résultats de l'apprentissage, mais également un lien remarquable avec l'enseignant lui-même, le programme suivi, le processus d'apprentissage, les méthodes utilisées, les approches et les techniques, ainsi que les ressources dans leur ensemble.

Tout au long du processus de l'enseignement, c'est l'enseignant qui joue un rôle primordial pour assurer un prolongement et une cohérence avec les apprenants pour qu'ils puissent organiser leurs actions pédagogiques. Le processus d'évaluation dans l'enseignement des langues étrangères agrège différents objectifs. Les questions importantes auxquelles il convient de répondre au cours du processus d'évaluation peuvent être énumérées comme suit :

- « De définition du concept d'évaluation (qu'évaluer?);

- De cibles, d'objets de l'évaluation (quoi évaluer?);

- De fonctions, d'objectifs et de buts de l'évaluation (pour quoi évaluer?)

- De temps, de moment d'application de l'évaluation (quand évaluer ?);

- De procédure de l'évaluation (comment évaluer ?);

- De destinataires de l'évaluation (pour qui évaluer?); 
- Des auteurs de l'évaluation (qui évalue ?).» (Mboup, 2003, pp. 57-58).

A partir de cette citation, on peut dire que le concept d'évaluation possède différentes significations interdisciplinaires en termes de détermination des objectifs.

Dans le contexte de l'enseignement de LE, l'évaluation des aptitudes à l'expression écrite peut être définie en fonction de la manière dont les aptitudes à l'écriture des élèves se reflètent dans leurs performances. À cet égard, la question de « quoi évaluer?» se situe dans la première étape.

Cette évaluation peut être la détermination des attitudes, motivations ou niveaux de compétence de production écrite des apprenants. Les stratégies utilisées dans les activités d'écriture ou dans le programme suivi peuvent être considérées comme une autre variable. Parce que l'écriture est un processus complexe et ce processus est influencé par différentes variables. Dans la deuxième étape, la réponse à la question « pourquoi évaluer?» est recherchée. A ce stade, les objectifs, les buts et les fonctionnalités de l'évaluation sont mis en évidence. Au cours de ce processus, un test de diagnostic est exécuté pour déterminer les niveaux des apprenants et vérifier les informations préliminaires. Afin de connaître les étapes de développement du processus et de disposer d'informations sur ses faiblesses ou ses points forts, une évaluation du processus est effectuée, ainsi qu'une évaluation sommative afin de déterminer si les objectifs souhaités sont atteints.

La question de « quand évaluer ? » peut-être variée en fonction du programme suivi, du type d'activités d'écriture, des stratégies utilisées et du niveau des apprenants. La manière dont cette évaluation est effectuée est très importante. Essentiellement, les études quantitatives et qualitatives sont effectuées pour évaluer la compétence de production écrite. Alors que les données qualitatives montrent les attitudes et les motivations des apprenants à l'écriture et leur capacité à écrire des textes, les données quantitatives sont examinées comme un processus de collecte de données concrets permettant un contrôle progressif du processus.

Diverses échelles et rubriques sont utilisées pour collecter des données qualitatives et quantitatives au cours du processus d'évaluation. Dans les méthodes traditionnelles, l'évaluation ne se fait généralement qu'avec des examens, tandis que les nouveaux modèles d'enseignement et d'apprentissage utilisent de différentes méthodes, telles que l'évaluation par projet, l'évaluation par les pairs, l'évaluation des activités en classe et parascolaires et l'évaluation de portfolio. Surtout, avec l'approche communicative, la dimension de communication de la langue s'est changée. Par conséquent le processus de communication des apprenants d'une LE est évalué avec de nombreuses variables. Dans le modèle de perspective actionnelle, l'utilisation active de la langue par les apprenants en tant qu'acteur social dans chaque environnement donne une importance différente au processus d'évaluation.

\subsection{Evaluation Numérique et Développement de la Production Ecrite chez les Apprenants de FLE}

A l'époque actuelle où les technologies de l'information et de la communication sont intégrées pas à pas dans tous les domaines, les établissements scolaires ont connu une mutation sur le renouvèlement des pratiques d'évaluation. Pour recueillir les informations et synthétiser les acquis, l'enseignant peut différencier et utiliser les opportunités offertes par le numérique afin de mieux soutenir les apprentissages individuels.

La multiplicité des plateformes numériques y compris les outils, les logiciels, les supports et ainsi toutes sortes de médias a renouvelé l'évaluation. Pour améliorer les stratégies d'évaluation dans les établissements scolaires, le numérique peut apporter des avantages considérables et ainsi peut répondre à divers objectifs dans le cadre d'évaluation des compétences et d'auto-évaluation.

Le numérique offre aux enseignants la possibilité de favoriser l'interaction avec les apprenants et de mobiliser les savoirs pour créer un réel continuum en dehors de l'école. 
«La communication synchronique peut être menée dans un esprit conversationnel comme la pratique du téléphone ou la communication à titre d'exemple via Skype quant à la communication asynchronique, elle est réalisée à travers la discussion sur les forums ou l'envoi des messages par le courriel électronique. » (Kandeel, 2014, p. 37).

Par le numérique, les nouvelles modalités d'évaluation ont été créées et adaptées aux besoins des apprenants dans le meilleur suivi de leurs progressions au contexte pédagogique. « Les progrès technologiques réalisés ces vingt dernières années ont non seulement favorisé l'intégration de contenus multimédias et le développement de nouveaux formats d'items, mais ont aussi permis d'envisager de nouvelles possibilités d'interaction et de correction automatique.» (Burton, R., Reichert, M., \& Martin, R., 2009, p. 58). Ce qui est important à ce stade, c'est de s'assurer de l'efficience et de l'efficacité du numérique comme soutien au parcours de formation.

Evaluer à l'ère du numérique est une tendance nouvelle et un phénomène récent. Le progrès de la technologie éducative a facilité l'échange entre les enseignants et les apprenants. Par conséquent, la démarche d'évaluation peut s'entraîner non seulement à l'école, mais aussi en dehors de l'école. D'après le concept d'évaluation numérique, ce que nous comprenons, c'est que les examens et les évaluations ont lieu dans des environnements numériques, par opposition à l'évaluation traditionnelle. Bien que seuls les examens à choix multiples puissent être évalués à l'avance, le développement et la fonctionnalité des plates-formes numériques et des outils multimédias de nos jours permettent d'évaluer toutes les compétences linguistiques. L'un des premiers exemples de plateformes d'évaluation numérique est le module Dialang soutenu par la Commission européenne.

« Dialang est le premier système d'EAO majeur mis gratuitement à disposition sur internet. Il a été conçu à partir du Cadre européen commun de référence pour les langues. Il a été développé par plus de vingt universités européennes sous la tutelle de la Commission européenne. Il permet de réaliser des évaluations formatives équivalentes dans quatorze langues de l'Union européenne pour l'écoute, la lecture, la rédaction, la grammaire et le vocabulaire. » (Burton et al.,2009, p. 59).

Ce module offre la possibilité de faire une évaluation de processus pour les compétences langagières. Les activités du module sont préparées sous la forme d'un examen et les commentaires sont obtenus rapidement à la suite de chaque activité. Il est possible de dire que les apprenants de langues ont l'occasion de voir leurs propres niveaux dans le contexte de l'autoévaluation. En premier, un test de diagnostic est effectué pour voir le niveau de connaissance des apprenants. Ce test met l'accent sur la capacité de vocabulaire. Ce module est accessible via Internet ou téléchargé sur des ordinateurs personnels. C'est un module gratuit et facile à utiliser qui est plus utile dans le contexte du développement des compétences et de l'auto-évaluation. A la suite des activités, une évaluation détaillée est réalisée et des informations sont fournies sur le statut actuel d'apprentissage des apprenants. Des modules similaires sont facilement accessibles via Internet.

En particulier pour la compétence de grammaire et d'orthographe, les apprenants peuvent accéder aux divers modules en termes d'évaluation. Les correcteurs en ligne sont très populaires et utilisés largement par les apprenants de LE. Les correcteurs en ligne permettent aux apprenants de contrôler, de réviser les erreurs syntaxiques et/ou orthographiques dont l'objectif est de soutenir le processus de production écrite comme la révision. « Les correcteurs syntaxiques dont sont aujourd'hui pourvus tous les traitements de texte constituent également une aide à la révision. » (Mangenot, 2012, p. 108). 


\section{CADRE METHODOLOGIQUE}

\subsection{Objectif de la Recherche}

Notre objectif principal dans cette recherche, est de mettre en évidence les effets et les limites d'évaluation numérique lors de développement et d'amélioration de la compétence de production écrite chez les étudiants qui apprennent le français comme LE. Nous avons pour objectif d'examiner l'influence des plateformes numériques par rapport à la méthode d'évaluation traditionnelle (papier-crayon) sur le processus de manuscrit.

\subsection{Méthode de la Recherche}

Dans notre étude, nous avons adapté le modèle de recherche-action des méthodes de recherche qualitative. On définit la recherche-action comme suit :

«...une approche systématique de la recherche qui permet aux chercheurs de trouver des solutions efficaces aux problèmes auxquels ils sont confrontés dans leur vie quotidienne. Contrairement à la recherche expérimentale ou scientifique traditionnelle qui cherche des justifications généralisables qui pourraient s'appliquer à tous les contextes, la rechercheaction se concentre sur des situations spécifiques et des solutions localisées. » (Stringer, 2007, p. 1).

\subsection{Importance de la Recherche}

Cette recherche vise à construire un modèle alternatif à l'évaluation de la production écrite. Nous pouvons énumérer les principaux avantages comme les suivants :

1. Le correcteur peut corriger les copies des examens écrits n'importe quand et n'importe où sur un ordinateur et ainsi sur une tablette connectée au web,

2. Une fois les critères ainsi que les barèmes de l'évaluation sont intégrés dans la plateforme numérique, l'exécution est vraiment rapide pour les correcteurs,

3. Le correcteur a des possibilités de marquer sur les copies en lignes, de commenter, d'écrire, de donner des feedbacks de manière immédiate et ainsi de noter à l'écran,

4. Grâce à la plate-forme numérique, les étudiants peuvent accéder, de manière synchrone ou asynchrone, aux résultats des activités accomplies pour qu'ils puissent voir les rétroactions plus détaillées afin de recueillir les informations sur les points forts et sur les points faibles,

5. Les étudiants peuvent construire leurs portfolios numériques pour porter un jugement sur la valeur de leur développement et pour contrôler le processus d'amélioration de la compétence de production écrite.

\section{ANALYSE DES DONNEES}

Dans cette partie, nous avons analysé les données obtenues à la fois qualitatives et quantitatives suite à la recherche. Cette partie se compose de trois sous-parties. Dans la première sous-partie, nous avons traité les notes moyennes de Test Préliminaire 1 et de Test Final 1 des étudiants en compétence d'écriture. Dans la deuxième, nous avons analysé les données concernant les notes moyennes de Test Préliminaire 2 et de Final 2. Dans la troisième sous-partie, nous avons traité les notes moyennes de Test Préliminaire 3 et de Final 3.

\subsection{Comparaison de Test Préliminaire 1 et de Test Final 1}

Dans cette partie, nous avons analysé les données relatives aux capacités à présenter/exprimer des faits, des évènements dans le cadre de cohérence et de cohésion des copies des étudiants. 
En ce qui concerne la première partie de la grille d'évaluation le tableau ci-dessous représente les notes moyennes du Test Préliminaire 1 et du Test Final 1.

Tablo 1. Test sur Échantillon Unique de Test Préliminaire 1 et de Test Final 1

Statistiques sur Échantillon Unique

\begin{tabular}{|c|c|c|c|}
\hline $\mathrm{N}$ & Moyenne & Ecart-Type & Erreur Standard Moyenne \\
\hline Test Préliminaire1 28 & 7,714 & 2,5255 & ,4773 \\
\hline Test Final 1 & 10,679 & ,9251 & , 1748 \\
\hline
\end{tabular}

Test sur Échantillon Unique

Valeur du test $=0$

\begin{tabular}{llllll} 
& & & & & \multicolumn{2}{l}{$\begin{array}{l}\text { Intervalle de Confiance } \\
\text { Différence }\end{array}$} & $\begin{array}{l}\text { 95\% de la Différence } \\
\text { Inférieure }\end{array}$ & Supérieure \\
\hline Test Préliminaire1 & 16,16327 &, 000 & 7,7143 & 6,735 & 8,694 \\
Test Final1 & 61,08027 &, 000 & 10,6786 & 10,320 & 11,037 \\
\hline
\end{tabular}

Dans l'échelle d'évaluation, le score maximum que les étudiants pouvaient obtenir pour cette partie était de 13 points. A partir des données recueillies, nous avons vu que la moyenne la plus basse était de 6,7 sur 13 et la plus haute était de 8,6 sur 13 pour le Test Préliminaire 1 et concernant le Test final 1; la moyenne la plus basse était de 10,3 et la moyenne la plus haute était de 11 sur 13. Cela a montré que les étudiants ont fait de grands progrès au Test Final 1 par rapport aux résultats du Test Préliminaire 1 . Le point $\mathrm{P}$ a été calculé comme , $000<0,05$ et cela a montré qu'il y avait une différence significative en faveur du groupe expérimental. Alors que le score moyen du Test Préliminaire était de 7 points, cette moyenne a augmenté de 3 points de plus puisque la moyenne de Test Final était de 10 points.

La première partie de la Grille d'Evaluation comprend quatre dimensions. La première met l'accent sur le Respect de la Consigne dont l'objectif est d'évaluer les copies des étudiants dans le cadre de leur capacité de suivre les consignes. Nous avons observé que les étudiants n'avaient pas suffisamment suivi cet élément dans les activités des deux premières semaines. Ils se sont concentrés sur un mot différent dans les consignes et c'est pour cette raison que l'intégrité du texte n'a pas été prise en compte. Notre première intervention conformément à la rechercheaction, était de fournir aux étudiants un exemple d'analyse de texte.

Le deuxième élément de la Grille d'Evaluation représente la Capacité à Présenter des Evénements. L'un des défis les plus importants pour les étudiants était que leur connaissance de vocabulaire n'était pas suffisante. Nous pouvons dire que les étudiants ont souvent du mal à décrire les évènements ou à faire des descriptions.

Le troisième élément comprend la Capacité à Exprimer sa Pensée. D'après cet élément, on attend que les étudiants puissent exprimer leurs sentiments et donner leurs avis sur le sujet traité. L'un des problèmes les plus courants en particulier était que, même si les étudiants avaient suffisamment parlé de l'importance d'un sujet, ils ne pouvaient pas présenter leurs idées de manière concrète.

Le dernier élément de la rubrique met l'accent sur la Cohérence et Cohésion de texte auquel la majorité des étudiants ont confronté les difficultés au cours de la réalisation des activités. Nous avons également constaté que les connecteurs nécessaires n'avaient pas été utilisés suffisamment pour que les phrases soient complètes.

\subsection{Comparaison de Test Préliminaire 2 et de Test Final 2}

Dans cette partie, nous avons analysé les données concernant le développement de la compétence du vocabulaire. Le tableau ci-dessous relatif à la deuxième partie de la grille d'évaluation représente les notes moyennes de Test Préliminaire 2 et de Final 2. 
Tablo 2. Test sur Échantillon Unique de Test Préliminaire 2 et de Test Final 2

Statistiques sur Échantillon Unique

\begin{tabular}{lllll}
\hline & $\mathrm{N}$ & Moyenne & Ecart-Type & Erreur Standard Moyenne \\
\hline Test Préliminaire2 & 28 & 2,857 & 1,0440 &, 1973 \\
Test Final2 & 28 & 4,286 &, 5516 &, 1042
\end{tabular}

Test sur Échantillon Unique

Valeur du Test $=0$

\begin{tabular}{lllllll} 
& $\mathrm{t}$ & $\mathrm{ddl}$ & $\begin{array}{l}\text { Sig. } \\
\text { (Bilatérale) }\end{array}$ & $\begin{array}{c}\text { Différence } \\
\text { Moyenne }\end{array}$ & $\begin{array}{l}\text { Intervalle de Confiance } \\
95 \% \text { de la Différence } \\
\text { Inférieure }\end{array}$ & Supérieure \\
\hline Test Préliminaire2 & 14,481 & 27 &, 000 & 2,8571 & 2,452 & 3,262 \\
Test Final2 & 41,115 & 27 &, 000 & 4,2857 & 4,072 & 4,500 \\
\hline
\end{tabular}

Le Tableau 2 nous montre que dans le Test Préliminaire la moyenne de note la plus basse était de 2,4 sur 6 et la plus haute était de 3,2 sur 6. Concernant celle du Test Final; la moyenne la plus basse était de 4,0 et la moyenne la plus élevée était de 4,5 sur 6 . Selon les résultats de Test Final 2, nous avons vu un grand progrès dans la compétence lexicale et de l'orthographe chez les étudiants. Le point $\mathrm{P}$ a été calculé $000<0,05$ et cela a montré qu'il y avait une différence significative. Les analyses des copies ont montré que la majorité des étudiants se sont entendus sur la question du choix du vocabulaire pour exprimer les idées. Nous pouvons constater que deux types d'erreurs ont été fréquemment commises par les étudiants au cours des activités d'écriture. L'erreur de forme signifie l'erreur orthographique, alors que l'erreur de concept est l'utilisation de mots inappropriés dans le contexte. Dans la partie suivante, nous présenterons les exemples les plus fréquentes que se confrontent certains étudiants dans le Test Préliminaire 2. Il est possible de dire que les étudiants connaissent le sens de nombreux mots, mais ils n'utilisent pas suffisamment ces mots. On voit que les étudiants utilisent généralement des mots similaires. Dans les activités réalisées au cours de premières semaines, selon les fréquences des mots, nous pouvons dire que les étudiants utilisaient les mêmes mots dans les activités d'écriture. Bien qu'ils connaisent plusieurs mots, ils ne les utilisent pas activement tant à oral qu'à l'écrit. L'une des principales raisons pour lesquelles les élèves commettent de telles erreurs est de formuler les idées dans leur langue maternelle et d'essayer de les exprimer dans la langue cible. Ces erreurs, généralement supposées qu'elles proviennent d'un manque de vocabulaire, montrent que les étudiants connaissent réellement les sens des mots mais ne connaissent pas leur contexte. De plus, on constate que les étudiants ne font pas assez de recherches dans le dictionnaire et font peu d'exercices sur le lexique. On voit que certains étudiants n'utilisent que les mots utilisés quotidiennement dans les activités d'écriture et n'incluent pas suffisamment les exercices de synonyme et de dérivation. Dans ce contexte, afin d'éviter ce problème, nous avons tout d'abord proposé des dictionnaires interactifs communs aux étudiants et leur permettant de s'exercer dans le contexte de la recherche par dictionnaire. Lors de ces activités, nous avons conseillé aux étudiants de ne pas rechercher les équivalents turcs des mots, d'analyser les exemples de phrases des dictionnaires et de créer de nouvelles phrases en se concentrant sur les explications en français.

\subsection{Comparaison de Test Préliminaire 3 et de Test Final 3}

Le tableau 3 représente les résultats de troisième partie de la rubrique d'évaluation de la production écrite au niveau B1 au contexte de compétence grammaticale et orthographe grammaticale de Test Préliminaire 3 et de Test Final 3. 
Tablo 3. Test sur Echantillon Unique de Test Preliminaire 3 et de Test Final 3

Statistiques sur Echantillon Unique

\begin{tabular}{lllll}
\hline & $\mathrm{N}$ & Moyenne & Ecart-Type & Erreur Standard Moyenne \\
\hline Test Préliminaire3 & 28 & 3,054 & 1,0305 &, 1947 \\
Test Final3 & 28 & 4,071 &, 5040 &, 0952
\end{tabular}

Test sur Echantillon Unique

\begin{tabular}{lllllll}
\hline & \multicolumn{2}{l}{ Valeur du Test $=0$} & & Intervalle de Confiance \\
& $\mathrm{t}$ & ddl & $\begin{array}{l}\text { Sig. } \\
\text { (Bilatérale) }\end{array}$ & $\begin{array}{c}\text { Différence } \\
\text { Moyenne }\end{array}$ & $\begin{array}{c}\text { 95\% de la Différence } \\
\text { Inférieure }\end{array}$ & Supérieure \\
\hline Test Préliminaire3 & 15,680 & 27 &, 000 & 3,0536 & 2,654 & 3,453 \\
Test Final3 & 42,750 & 27 &, 000 & 4,0714 & 3,876 & 4,267 \\
\hline
\end{tabular}

Selon les résultats de deux Tests, nous voyons que la moyenne de note la plus basse était de 2,6 sur 6 et la plus haute était de 3,4 sur 6 et celle de Test Final; la moyenne la plus basse était de 3,8 et la moyenne la plus haute était de 4,2 sur 6 . A partir des résultats de Test Final 2, nous avons constaté que les étudiants avaient fait de grands progrès dans la compétence grammaticale et orthographe grammaticale. Le point $\mathrm{P}$ était $, 000<0,05$ et cela nous a montré qu'il y avait une différence significative.

La troisième partie de l'échelle d'évaluation, celle de la compétence grammaticale et orthographe grammaticale, comprend trois éléments au total et chaque élément vaut deux points. Le score le plus élevé est de six points que puissent les élèves obtenir dans cette section. Dès la première semaine, on constate que les scores des étudiants dans cette section sont assez faibles. Bien que les étudiants aient suivi cinq semestres de cours de grammaire au total à partir de classe préparatoire, nous pouvons constater qu'ils ont de sérieuses difficultés, en particulier en ce qui concerne la conjugaison, l'accord, le relatif et la préposition.

$\mathrm{Au}$ cours de la recherche, conformément au modèle de recherche-action, nous avons d'abord planifié d'identifier les natures de problèmes, de les classer et d'identifier les forces et les faiblesses des étudiants. "L'analyse des erreurs comporte un certain nombre d'étapes: recueillir des données, identifier les erreurs, classer les erreurs, quantifier les erreurs, analyser la source, remédier. » (Selinker \& Gass, 2008, p. 103). Pour ce faire, nous avons classifié les types d'erreurs commises par les étudiants au Test Préliminaire en donnant les fréquences de ceux-ci dans le tableau ci-dessous ;

Tablo 4. Types d'Erreurs

\begin{tabular}{lcc}
\hline Types d'Erreurs & Test Préliminaire 3 & Test Final 3 \\
\hline & Fréquence & Fréquence \\
Article & 15 & 8 \\
Quantitatif & 15 & 11 \\
Démonstratif & 10 & 6 \\
Pronoms personnels & 20 & 11 \\
Relatif & 16 & 9 \\
Possessif & 8 & 1 \\
Préposition & 46 & 34 \\
Auxiliaires & 12 & 4 \\
Accord & 83 & 41 \\
Total & 225 & 125 \\
\hline
\end{tabular}


A partir de ce tableau, 225 erreurs ont été faites au total dans le test préliminaire au niveau de grammaire et d'orthographe. Nous voyons que le pourcentage le plus élevé est lié à l'accord dont la fréquence est de 83. Le deuxième pourcentage le plus élevé appartient à la préposition et en ce qui concerne le possessif, la fréquence la plus basse est de huit au total. A la suite des résultats obtenus, nous avons d'abord fourni aux étudiants des informations détaillées sur les types d'erreurs qu'ils commettaient fréquemment. En particulier, nous avons fourni aux étudiants les exemples d'erreurs dues au manque d'attention dans leurs propres rapports. Nous avons conseillé aussi aux étudiants de s'exercer sur divers sujets à partir d'exemples en partageant une variété de ressources numériques pour pratiquer et respecter les règles de grammaire et d'orthographe.

\section{CONCLUSION ET DISCUSSION}

Les données quantitatives de l'étude ont été utilisées pour mesurer le développement de la compétence de production écrite des étudiants. La première hypothèse de l'étude est la suivante :

Hypothèse 1: L'évaluation numérique favoriserait le progrès de la compétence de production écrite des étudiants.

A propos de la première hypothèse, quand les scores moyens obtenus lors du pré-test $(\bar{x}=13,5)$ et du post-test $(\bar{x}=19)$ des étudiants ont été comparés, une augmentation significative a été observée en faveur du post-test $(\mathrm{p}=, 000<0,05)$. Les performances de production écrite des étudiants ont été divisées en trois catégories et le processus de développement de chaque catégorie a été examiné séparément. Dans la première partie de ces sous-catégories, nous avons cherché à analyser le travail effectué à propos de la cohérence des textes et des phrases construites par les étudiants. Suite à l'analyse, lorsque les scores moyens des étudiants du pré-test $(\bar{x}=7,7)$ ont été évalués en termes de cohérence et de cohésion, nous avons remarqué une augmentation positive en faveur du post-test $(\bar{x}=10,6)$. Lors du processus de production de texte les problèmes les plus importants rencontrés par les étudiants étaient liés à la cohérence/cohésion. Les données obtenues par des pré-tests et dans les formulaires d'auto-évaluation des étudiants le montre clairement. Les résultats obtenus lors du post-test ont révélé que les étudiants surmontaient ce problème dans le processus de production des textes.

La deuxième partie à analyser, à la suite de résultats obtenus dans la partie cohérence et cohésion, porte sur le niveau de compétence lexicale/orthographe atteint par les étudiants et cela dans le but de vérifier s'il y a accord entre les deux. A partir de l'analyse, les moyennes des étudiants du pré-test $(\bar{x}=2,8)$ et du post-test $(\bar{x}=4,2)$ ont été comparées et l'augmentation des niveaux de compétence lexical/orthographe a été vérifiée. L'augmentation positive en faveur du post-test a également contribué de manière positive au développement de la compétence écrite des étudiants. De plus, le fait que l'augmentation de la première sous-section soit parallèle à celle de la deuxième sous-section augmente la validité des données. Dans la troisième sous-catégorie, les processus d'écriture des étudiants ont été discutés en termes de compétence grammaticale et d'orthographe grammaticale. À la suite de l'analyse, lorsque les scores moyens des étudiants du pré-test $(\bar{x}=3,0)$ ont été évalués en termes de compétence grammaticale/orthographe grammaticale, nous avons remarqué une augmentation positive en faveur du post-test $(\bar{x}=4,0)$.

Quand les niveaux de compétence grammaticale des étudiants ont été comparés aux niveaux de compétence lexicale et de cohérence textuelle, nous avons vu que l'augmentation des niveaux de compétence grammaticale n'était pas aussi significative que celle des deux autres niveaux, mais que cette augmentation était statistiquement positive. De même, dans leur travail, Grégoire et Karsenti (2013) concluent qu'il y a une augmentation en termes de vocabulaire et de cohérence/cohésion à l'exception de compétence grammaticale comme le montre notre travail. Ces résultats affirment également nos résultats. La compétence de production écrite des étudiants a été discutée en trois sous-catégories et il en a été conclu que les étudiants avaient progressé dans les trois sous-catégories qui soutenaient le processus de production écrite. La ressemblance et 
l'adéquation des résultats au processus d'évaluation de l'écriture divisé en trois sous-catégories montrent également que l'outil de mesure a une cohérence interne.

Hypothèse 2: L'usage de plateforme numérique permettra d'accroître la performance des étudiants en production écrite à l'aide de rétroaction immédiate de manière plus détaillée.

Les scores d'activité d'écriture effectués au cours de la première semaine constituent les données du pré-test de l'étude. La même activité d'écriture a été refaite lors de la 13ème semaine et les résultats ont constitué les données du post-test de l'étude. A la suite des résultats obtenus, en comparant les scores du pré-test et du post-test, le modèle de rétroaction instantanée et détaillée utilisant des outils d'évaluation numérique augmentait les performances des étudiants en écriture. Lorsque les scores totaux obtenus lors des travaux de rédaction antérieurs à l'étude ont été comparés au résultat total obtenu lors de travaux d'écriture postérieurs à l'étude, il y a eu une augmentation significative en faveur du post-test. Les scores obtenus lors des travaux d'écriture des étudiants ont été évalués individuellement, nous avons remarqué qu'il y avait une augmentation positive de la moyenne de 21 étudiants, alors qu'il n'y avait pas d'augmentation significative de la moyenne de deux étudiants.

Suite à l'évaluation des deux étudiants, les pré-tests étaient plus élevés que ceux des autres étudiants. Par conséquent, les scores de post-test des deux étudiants ont diminué par rapport aux scores de pré-test. Cependant, étant donné que l'étude a duré 13 semaines, nous avons constaté que les performances en écriture s'amélioraient certaines semaines mais d'autres semaines, en revanche, pouvaient diminuer. L'augmentation des scores des cinq étudiants ayant réalisé des progrès entre $1 \%$ et $1,5 \%$ dans les évaluations effectuées avant et après l'étude n'est pas significative selon les résultats de SPSS. À la suite de l'analyse détaillée de cinq étudiants qui n'ont fait aucun progrès, nous avons remarqué que les étudiants n'avaient pas pris en compte les commentaires reçus et n'avaient pas lu le matériel ni les avertissements partagés, en particulier dans les environnements d'évaluation numérique. Lors de l'analyse des documents d'écriture, il a été constaté que les performances d'écriture de 5 étudiants étaient supérieures à la moyenne, mais qu'ils ne pouvaient pas atteindre le niveau ciblé dans l'étude.

Quand les scores totaux ont été examinés semaine par semaine, les scores des étudiants avaient augmenté les $2^{\text {ème }}, 3^{\text {ème }}$ et $4^{\text {ème }}$ semaines, alors qu'ils avaient diminué de manière significative les $5^{\text {ème }}$ et $6^{\text {ème }}$ semaines. À la suite des entretiens menés avec l'enseignant et les étudiants, nous avons constaté que les étudiants manquaient de ressources pour améliorer leur écriture et que ce manque d'influence affectait leur motivation à écrire. Suite à l'intervention de deux heures effectuée avant la $7^{\text {ème }}$ semaine, nous avons déclaré que les étudiants seraient en mesure de partager des ressources dans un environnement numérique afin d'éliminer le manque de ressources. Cette intervention a eu un impact positif sur les performances des étudiants à la $7^{\text {ème }}$ semaine et les scores des étudiants ont augmenté. La réussite des étudiants en écriture s'est poursuivie jusqu'à la fin de l'étude. De même, dans une étude menée par Tezci et Dikici (2006), il a été constaté que l'évaluation numérique augmentait positivement le succès des étudiants en écriture.

Hypothèse 4: L'évaluation numérique contribue à l'augmentation des notes des examens partiels et des examens finaux.

Nous avons remarqué que l'évaluation numérique augmentait considérablement la réussite dans les examens partiels et finaux des étudiants. Afin de trouver la différence significative, les moyennes des examens du cours d'écriture ont été posées à l'instructeur concerné et comparées aux moyennes des examens de la période d'étude. À la suite de la comparaison, nous avons vu que la moyenne des examens partiels pour la période précédente était de 53,82 et qu'après l'étude, elle était passée à 71,64. L'augmentation entre les deux examens est de 33,6\%. De même, lorsque les moyennes finales sont prises en compte, les moyennes finales avant l'étude sont passées de 58,07 à 77,61 après l'étude. Le montant de l'augmentation est directement proportionnel à l'augmentation des notes des examens partiels. En outre, à la suite d'entretiens avec les étudiants 
et les enseignants, nous avons conclu que les résultats des étudiants avaient augmenté. Par conséquent, nous avons constaté qu'au lieu d'évaluer les compétences en écriture, l'évaluation en tant que processus évite la fossilisation des connaissances et des compétences des étudiants dans le processus d'apprentissage. Les erreurs ont été rapidement corrigées, notamment avec les supports numériques. Ces commentaires ont permis aux étudiants de reconnaître leurs erreurs dans le processus d'écriture et de les corriger avant qu'il ne soit trop tard. À la suite des données quantitatives obtenues, les étudiants ont montré des améliorations dans les parties de cohérence/cohésion, de compétence lexicale/orthographe et de compétence grammaticale/orthographe.

En ce qui concerne les données qualitatives, à la lumière des données obtenues, nous avons vu que les étudiants ont développé leur compétence de production écrite d'une manière significative.

Hypothèse 3 : Les portfolios numériques permettront aux étudiants de suivre leurs points forts et leurs points faibles.

Nous avons conclu que le portfolio permettait aux étudiants de voir leurs forces et leurs faiblesses. En particulier, les étudiants qui ont des difficultés à utiliser le temps imparti à l'examen ont indiqué qu'ils surmontaient ce problème grâce à une écriture régulière chaque semaine. Grâce aux commentaires fournis, les élèves ont pu se rendre compte des erreurs qu'ils commettaient fréquemment et ont donc pu les corriger. Par conséquent, nous avons remarqué que les notes obtenues à la compétence de production écirte n'avaient aucun sens pour eux avant l'étude car ils ne donnaient aucune information sur les erreurs commises et ne contribuaient pas à leur développement parce qu'ils ne montraient pas la correction qui empêche la méconnaissance des règles en français comme indiqué par YücelsinTaş, Candemir et Kara (2016, p.98). Les portfolios numériques, où les performances d'écriture des élèves sont enregistrées et stockées chaque semaine, ont permis aux étudiants de s'autoévaluer. En particulier, les étudiants qui comprennent leurs faiblesses cherchent l'autonomie pour trouver leurs propres faiblesses, ce qui les rend autonomes.

Quand les résultats quantitatifs et qualitatifs obtenus à partir de l'étude sont généralisés, on peut dire que l'évaluation numérique a un effet positif sur la compétence de production écrite des étudiants. Les résultats obtenus lors des activités d'écriture d'une durée de 13 semaines indiquent que $21(75 \%)$ des 28 étudiants ayant participé à l'étude ont atteint le niveau de réalisation souhaité et $5(17 \%)$ sont restés inchangés. Deux étudiants $(8 \%)$ dont les pré-tests étaient plus élevés que les post-tests ont montré une diminution. À la fin de l'étude, les étudiants et les enseignants ont déclaré que les commentaires formulés pendant et après le processus d'écriture augmentaient la motivation et le succès dans l'écriture.

\section{BIBLIOGRAPHIE}

Burton, R., Reichert, M., \& Martin, R. (2009). L'évaluation des compétences langagières. Dans J.-G. Blais, Evaluation des apprentissages et technologies de l'information et de la communication (p. 59). Québec: Les Presses de l'Université de Laval.

Dubois, J., Giacomo, M., Guespin, L., Marcellesi, C., Marcellesi, J.-B., \& Mével, J.-P. (2002). Dictionnaire de linguistique. Montréal: Larousse.

Glopper, K., Kruiningen, J., \& Hemmen, N. (2014). Context in Writing Process Research. Dans D. Knorr, C. Heine, \& J. Engberg, Methods in writing process research (p. 15). Frankfurt: Peter Lang Edition.

Grabowski, J. (1996). Writing and speaking: common grounds and differences toward a regulation theory of written language production. Dans C. Levy, \& S. Ransdell, The science of writing (p. 75). New Jersey: Lawrence Erlbaum Associates. 
Grégoire, P., \& Karsenti, T. (2013). Le traitement de texte et la qualité de l'écriture d'élèves québécois du secondaire. Education et Formation, 298(3), 9-28.

Kandeel, R. (2014). Les TIC dans les pratiques des enseignants du FLE pour la préparation du DELF. Frantice.net, 9, 31-53.

Karsenti, T., Savoie Zajc, L., \& Larose, F. (2001). Les futurs enseignants confrontés aux TIC: changements dans l'attitude, la motivation et les pratiques pédagogiques. Education et francophonie, 29(1), 86-124.

Mangenot, F. (2012). Ecrire avec l'ordinateur: du traitement de texte au web social. Le Français dans le Monde, Recherches \& Applications, 51, 107-117.

Mboup, M. (2003). Evaluation des acquis scolaires. Université Cheikh Anta Diop de Dakar, Ecole Normale Supérieur, 1-112.

Selinker, L., \& Gass, S. (2008). Second language acquisition. New York: Taylor \& Francis.

Stringer, E. (2007). Action research. London: Sage Publications.

Tezci, E., \& Dikici, A. (2006). The effects of digital portfolio assessment process on students' writing and drawing performances. The Turkish Online Journal of Educational Technology, 5(2), 46-55.

Yücelsin Taş, Y., Candemir, C., \& Kara, Ö. (2016). Interférences lexicales: cas de l'université de Marmara. Istanbul Journal of Innovation in Education, 2(3-2), 89-100.

\section{GENIŞLETILMIŞ ÖZET}

Yazma, temelinde dört alt beceriye ayrılan yabancı dil öğretiminin önemli bir bileşenidir. Hem öğrenen açısından geliştirilmesi hem de öğreten açısından değerlendirilmesi oldukça zor bir süreç olarak görülmektedir. Bu süreçte öğretenlerin yazma süreçleri ve bu süreçlerde yapılan hatalar konusunda öğrenenlere farkındalık kazandırması beklenir. Gelişim aşamasındaki yazma becerilerine verilen dönütler ve düzeltmeler sistematik yapılırsa öğrenenlerin yazma becerileri gelişebilir ve hataların kalıcılığı önlenebilir.

Özellikle 21. yüzyılın teknolojik alt yapısı düşünüldüğünde belki de yazma becerilerine verilebilecek en faydalı dönütün çevrimiçi ortamlar olduğu düşünülebilir. Bu bağlamda yazma becerilerinin değerlendirilmesinde teknoloji bütünleşme sürecinin gerekli olduğu söylenebilir. Dijital platformlar aracılığıyla özellikle yazma becerisinin geliştirilmesine yönelik gerçekleştirilen etkinliklerin değerlendirilmesi ve dönütlerin hızlı bir şekilde öğrenenlere iletilmesi kolaylaşmıştır. Yaygın olarak kullanılan dijital değerlendirme araçlarının geleneksel değerlendirme yöntemine kıyasla üstünlüklerinden bahsetmek mümkündür. Bu çalışmada pek çok eğitim kurumu tarafından etkin bir şekilde kullanılan Gradescope uygulamasından yararlanılmıştır. Özellikle alanyazında sıklıkla vurgulandığı üzere, dijital araçların kullanımına yönelik hem öğrencilerin hem de öğretmenlerin süreç öncesinde bilgilenmesi değerlendirme etkinlikleri boyunca karşılaşılabilecek olası sorunlara karşı önemli bir göstergedir. Bu çalışmada teknoloji kullanımı ve teknoloji entegrasyonu arasındaki temel farklar sıklıkla vurgulanarak, olası teknik sorunlara karşı etkilerinden bahsedilmiştir. Anlık bir eylemi ifade eden teknoloji kullanımı kavramı ve teknoloji kullanım sürecinde gerekli teknik altyapının yeterli olmaması ve öğretmen ve öğrencilerin sistemin kullanımına yönelik bilgi eksiklikleri sürece olumsuz açıdan etki etmektedir. Teknoloji entegrasyonu ise teknoloji kullanımı kavramına kıyasla daha sistematik bir süreci ifade ederken; hem öğretmenlerin hem de öğrenenlerin süreç öncesinde ve süreç boyunca bilgilendirilmelerini hedeflemektedir. Bu nedenle, çalışma öncesi öğrencilere sistemin teknik yapısı, arayüz kullanımı ve değerlendirme süreci ile ilgili detaylı bilgilendirme yapılmış, uygulama sürecine geçilmeden önce öğrencilerin de sistemin yapısını etkin bir şekilde 
kavrayabilmeleri için deneme etkinlikleri gerçekleştirilmiştir. Bu sayede çalışma sürecinde olası aksaklıkların ve sorunların önüne geçilmeye çalışılmıştır.

Dijital değerlendirme araçlarının en önemli katkılarından birisi de öğrencilere hızlı dönüt sağlamak ve etkileşimli kaynakları sistem aracılığıyla sunabilmektir. Yazma etkinliklerine yönelik detaylı rapor alan öğrenciler, aynı zamanda kendilerine sunulan etkileşimli kaynaklar aracılığıyla bireysel öğrenme ve bireysel değerlendirme süreçlerine de katkı sunmuşlardır. Süreç değerlendirmesinin etkin olarak uygulandığı bu çalışma boyunca, öğrencilere değerlendirme maddelerinden aldıkları puan değerleri de iletilmiştir. Haftalık düzenli olarak gelişim süreçlerini takip eden öğrenciler aynı zamanda eksik oldukları alanları da kontrol etme olanăg da bulmuşlardır. Değerlendirme maddelerinde herhangi bir gelişim görülmemesi ya da istenilen düzeyde ilerleme sağlanamaması durumunda eylem araştırması modeline uygun olarak gerekli müdahaleler yapılmış, öğrencilerin görüşlerine de yer verilmiştir.

$\mathrm{Bu}$ çalışmanın temel amacı dijital değerlendirme araçlarının ve detaylı dönüt verme modelinin yabancı dil olarak Fransızca eğitiminde öğrencilerin yazma becerilerini geliştirmeleri üzerindeki etkilerini incelemektir.

Eylem araştırması modeline uygun olarak gerçekleştirilen bu araştırmada şu sorulara yanıt aranmıştır: Dijital değerlendirme araçlarının öğrencilerin Fransızca yazma becerilerini geliştirmeleri üzerindeki etkileri nelerdir? Dijital araçların değerlendirme sürecindeki rolü, katkıları, üstünlükleri ve sınırlılıkları nelerdir? Dijital değerlendirme ve detaylı dönüt verme modelinin yazma becerisinin geliştirilmesi sürecine etkileri nelerdir? Nitel ve nicel araştırma yöntemlerinin bir arada kullanıldığı bu çalışmanın evren ve örneklemini Ondokuz Mayıs Üniversitesi Yabanc1 Diller Eğitimi Bölümü Fransızca Öğretmenliği Anabilim Dalı'nda İleri Okuma ve Yazma dersine kayıtlı 28 öğrenci oluşturmaktadır.

Araştırmada yazma becerisine yönelik ön test, son test ve öz değerlendirme ölçeği kullanılmış ve elde edilen verilerin çözümlemesi SPSS paket programı kullanılarak hem nicel hem de nitel bulgular şeklinde yorumlanmıştır. 2018-2019 Eğitim Öğretim Yılı Bahar Döneminde gerçekleştirilen ve araştırma sonucunda elde edilen bulgulara göre, dijital değerlendirme araçlarının ve detaylı dönüt verme modelinin öğrencilerin Fransızca yazma becerilerini geliştirmede istatistiksel olarak anlamlı katkılar ortaya çıkardığı görülmüştür. Öğrencilerin ön test ve son test ortalamalarının karşılaştırılması sonucunda $P$ değeri, $000<005$ olarak hesaplanmış, bu değer deney grubunun lehine anlamlı bir sonuç ortaya çıtı̆̆ııı göstermiştir.

Çalışmadan elde edilen verilerin ve sunulan önerilerin, yabancı dil eğitiminde bilgi ve iletişim teknolojilerinin bütünleşme sürecine, özellikle değerlendirme boyutuna katkı sağlayacağ düşünülmektedir. Günümüz dijital dünyasında öğrenme ve öğretim boyutunun yanı sıra değerlendirme süreçlerinin de dijital otamlarda etkin bir şekilde yürütülebileceği yadsınamaz bir gerçektir. Özellikle bireysel değerlendirme süreçlerinin etkin bir şekilde yürütülebilmesi için dijital platformların eğitim kurumları tarafından yaygın olarak kullanıldığını söylemek mümkündür. Çalışmanın uygulama sürecinde öğrencilere yazma becerilerine yönelik CLE International yayınevi tarafından hazırlanan DELF B1 yazma aktiviteleri sunulmuş ve aynı zamanda öğrencilerin bu sınava hazırlanmasına da olanak sağlanmıştır. Çalışmamızda yararlandığımız dijital paltformun ve eşdeğer platformların da hem yazma becerisinin gelişimine hem de diğer becerilerin gelişim sürecine de katkı sağlayacağı düşünülmektedir. Özellikle dijital değerlendirme araçlarının temel bir eğitim alınarak beceri geliştirme etkinliklerinde aktif olarak kullanılmasının da önemi vurgulanmıştır. 